\title{
GENERALIZED LAURENT SERIES FOR SINGULAR SOLUTIONS OF ELLIPTIC PARTIAL DIFFERENTIAL EQUATIONS ${ }^{1}$
}

\author{
MURRAY WACHMAN
}

Introduction. Let $\bar{L}$ be a linear elliptic differential operator with analytic coefficients in a region $R$ of $E_{n}$. Let $L$ be the adjoint of $\bar{L}$. This paper extends the previous work of $\mathrm{F}$. John ${ }^{2}$ on representation of a solution $u$ of $\bar{L}[u]=0$, where $u$ has a singularity of finite order. A representation is developed here for a solution $v$ of $\bar{L}[v]=0$, where $v$ has an isolated essential singularity. This representation is a generalization of the Laurent series. Here the summation over the $n$th powers is replaced by summation over the $n$th derivatives of a fundamental solution $K(x, z)$, of the operator $L$. The representation in general is not unique.

Uniqueness of a suitably normalized representation is proved for the case in which $\bar{L}$ is homogeneous with constant coefficients. This gives rise to a theorem which for the three-dimensional Laplace operator reduces to the Maxwell-Sylvester theorem. ${ }^{8}$

The general case. Let $v(x)$ be a solution of $\bar{L}[v(x)]=0$, which has an isolated essential singularity at $x=y$, an interior point of the real region $R$, but is otherwise regular in the deleted region $R$. Let $\bar{L}$ be of order $m$.

Let $D \subset R$ be an open annular domain about the point $x=y$. Let $S_{1}$ be the sphere bounding the outer ball $B_{1}$ of $D$ and let $S_{2}$ be the sphere bounding the inner ball $B_{2}$ of $D$. Both $B_{1}$ and $B_{2}$ have the point $x=y$ as center and $B_{1} \neq B_{2}$. We further assume that $B_{1}$ is so small that $K(x, z)$, a fundamental solution of $L$, is analytic for $x \neq z$ in $B_{1}$.

ThEOREM I. For $z \in D, v(z)$ permits the following representation:

$$
v(z)=\omega(z)-\left.\sum_{\nu=0}^{\infty} \sum_{|i|=\gamma} A_{i} D^{i} K(x, z)\right|_{x=y},
$$

where $\omega(z)$ is analytic for $z \in B_{1}, A_{i}$ are constants depending on $S_{2}$, $|i|=i_{1}+i_{2}+\cdots+i_{n}$ and

Received by the editors December 4, 1962.

${ }^{1}$ I am indebted to Professor Fritz John for suggesting this problem.

2 See [1].

${ }^{3}$ See $[2$, pp. 514-521]. 


$$
D^{i}=\frac{\partial^{|i|}}{\partial x_{1}^{i_{1}} \partial x_{2}^{i_{2}} \cdots \partial x_{n}^{i_{n}}} .
$$

Proof. We know that there exists a ball $B_{3} C B_{2}$ with center at $x=y$ such that $K(x, z)$ has a Taylor series expansion with respect to $x$, about $x=y$, for $x \in B_{3}, z \in \mathcal{D}$. Let $S_{3}$ be the sphere bounding $B_{3}$ and let $\bar{R}$ be the annular region defined by $S_{1}$ and $S_{3} . \bar{R}$ has the boundary $\beta=S_{1} \cup S_{3}$. Applying Green's identity to the operator $\bar{L}$ over $\bar{R}$ we get for $z \in \mathbb{D}$ :

$$
\begin{aligned}
v(z) & =\int_{\beta} M[K(x, z), v(x)] d S_{x} \\
& =\int_{x \in S_{1}} M[K(x, z), v(x)] d S_{x}-\int_{x \in S_{3}} M[K(x, z), v(x)] d S_{x},
\end{aligned}
$$

where $M$ is a bilinear operator, and $d S_{x}$ denotes integration over the surface of the boundary.

$$
\omega(z)=\int_{x \in S_{1}} M[K(x, z), v(x)] d S_{x}
$$

is clearly analytic for $z \in D$, since $x \in S_{1}$ and therefore $x \neq z$.

For $z \in D, x \in S_{3}, K(x, z)$ has the Taylor series expansion about $x=y$ :

$$
K(x, z)=\sum_{\nu=0}^{\infty} \sum_{|i|=\nu} \frac{1}{i !}(x-y)^{i} D_{y}^{i} K(y, z),
$$

where $D_{y}^{i} K(y, z)=\left.D^{i} K(x, z)\right|_{x=y} ;(x-y)^{i}=\left(x_{1}-y_{1}\right)^{i_{1}} \cdots\left(x_{n}-y_{n}\right)^{i_{n}}$; and $i !=\left(i_{1} !\right) \cdots\left(i_{n} !\right)$. Equation (1) then becomes:

$$
v(z)=\omega(z)-\int_{x \in S_{3}} M\left[\sum_{\nu=0}^{\infty} \sum_{|i|=\nu} \frac{1}{i !}(x-y)^{i} D_{y}^{i} K(y, z), v(x)\right] d S_{x} .
$$

Since $M$ is a linear operator and the series is uniformly convergent:

$$
v(z)=\omega(z)-\sum_{\nu=0}^{\infty} \sum_{|i|=\nu} \frac{1}{i !} D_{y}^{i} K(y, z) \int_{x \in S_{z}} M\left[(x-y)^{i}, v(x)\right] d S_{x} .
$$

Let

$$
A_{i}=\frac{1}{i !} \int_{x \in S_{3}} M\left[(x-y)^{i}, v(x)\right] d S_{x} .
$$

$A_{i}$ is a constant. We then have as a final expression: 


$$
v(z)=\omega(z)-\sum_{v=0}^{\infty} \sum_{|i|=\nu} A_{i} D_{y}^{i} K(y, z) .
$$

It would be desirable to normalize equation (2), i.e., to obtain a form of equation (2) in which the $A_{i}$ are independent of the spheres $S_{2}$ and $S_{3}$. Equation (2) would then be unique and hold for any $z \neq y$, $z \in B_{1}$; because for any such $z$, we can find a $B_{2}$ and a $B_{3}$ with radius so small that the required Taylor series expansion for $K(x, z)$ will exist.

We shall succeed in giving such a normalization of equation (2) only for the special case of $\bar{L}$ homogeneous with constant coefficients, i.e.,

$$
\bar{L}=\sum_{|i|=m} b_{i} D^{i},
$$

where the $b_{i}$ are real constants. Let the coefficient of

$$
D^{I}=\frac{\partial^{m}}{\partial x_{1}^{m}}
$$

in $\bar{L}$ be $b_{I}$. Since $\bar{L}$ is elliptic $b_{I}$ is not zero. We know that there exists a fundamental solution of $\bar{L}$ of the form $K(y-x),{ }^{4}$ we shall use this fundamental solution in Theorem II.

THEOREM II. ${ }^{5}$ Let $v(x)$ be a solution of $\bar{L}[v(x)]=\sum_{|i|=m} b_{i} D^{i} v(x)=0$ for $x \in B_{1}, x \neq y$. Let $v$ have an isolated essential singularity at $x=y$. Then there exists a representation of $v(z)$ of the form:

$$
v(z)=\omega(z)-\sum_{y=0}^{\infty} \sum_{|s|=y} A_{s} D_{y}^{s} K(y-z), \quad \text { for } z \neq y, z \in B_{1},
$$

where $A_{s}=0$, whenever $D_{v}^{*}$ contains $D_{v}^{I}=\partial^{m} / \partial y_{1}^{m}$ as factor (i.e., whenever $\left.s_{1} \geqq m\right)$; and this representation is unique.

Proof. For $z \neq y, z \in D$, using equation (2) we can write:

$$
v(z)=\omega_{1}(z)-\sum_{\nu=0}^{\infty} \sum_{|k|=\nu} A_{k} D_{y}^{k}[K(y-z)] .
$$

We will prove that each operator term $\sum_{|k| \Rightarrow v} A_{k} D_{y}^{k}$ in the above series, with $\nu \geqq m$ can be written in the following form when it is applied to any solution $u$ of $\bar{L}[u]=0$.

${ }^{4}$ See [1, pp. 298-303].

5 This is a generalization of the Maxwell-Sylvester theorem for the case of the three-dimensional Laplacian. See [2, pp. 514-521]. 


$$
\sum_{|k|=>} A_{k} D^{k} u=\sum_{|\varepsilon|=\nu ; s_{1}<m} J_{8} D^{s} u
$$

where $J_{s}$ are constants. We will also prove that equation (5) is unique. From equation (3) we have:

$$
D^{I} u=\frac{1}{b_{I}}\left[\bar{L}-\sum_{|i|=m ; i \neq I} b_{i} D^{i}\right] u=-\sum_{|i|=m ; i \neq I} \frac{b_{i} D^{i} u}{b_{I}} .
$$

The operator term $\sum_{|k| \Rightarrow y} A_{k} D^{k}$ can be written as a polynomial in powers of $\partial / \partial x_{1}$, i.e.,

$$
\sum_{|k|=\nu} A_{k} D^{k}=\sum_{j=0 ; j+|\mu|=\gamma_{i} \mu_{1}=0}^{p} \sum_{\mu} G_{\mu+j} D^{\mu} \frac{\partial^{j}}{\partial x_{1}^{j}},
$$

where $G_{\mu+j}$ are constants, ${ }^{6} p$ a non-negative integer which is the highest order of $\partial / \partial x_{1}$ in $\sum_{|k|=\nu} A_{k} D^{k}$. For solutions $u$ of $\bar{L}[u]=0$ and for $p \geqq m$, using equation (6), we can reduce the $p$ th order polynomial operator in $\partial / \partial x_{1}$ in equation $(7)$ to a $(p-1)$ th order polynomial in $\partial / \partial x_{1}$. It is then seen that after $p-m+1$ steps equation (7), when applied to solutions $u$ of $\bar{L}[u]=0$ reduces to:

(8) $\sum_{|k|=>} A_{k} D^{k} u=\sum_{j=0 ; j+|\mu|=p_{i} \mu_{1}=0}^{m-1} \sum_{\mu} H_{\mu+j} D^{\mu} \frac{\partial^{j}}{\partial x_{1}^{j}} u=\sum_{|\ell|=p ; \delta_{1}<m} J_{\delta} D^{s} u$,

where $H_{\mu+j}, J_{s}$ are constants.

We now prove uniqueness. Suppose we had the two identities, for all $u$ with $\bar{L}[u]=0$ :

$$
\sum_{|k|=\nu} A_{k} D^{k} u=\sum_{|\varepsilon|=p_{i} \varepsilon_{1}<m} J_{s} D^{s} u
$$

and

$$
\sum_{|k|=0} A_{k} D^{k} u=\sum_{|\&|=v ; s_{1}<m} J_{l}^{\prime} D^{s} u
$$

then

$$
\hat{L}[u]=\sum_{|s|=v_{i} s_{1}<m}\left(J_{s}-J_{s}^{\prime}\right) D^{s} u=0 .
$$

Equation (10) holds for all solutions $u$ of $\bar{L}[u]=0$. Consider solutions $u$ of the form:

$$
u(x)=f\left(x_{1}\right) e^{\sigma_{2} x_{2}+\cdots+\sigma_{n} x_{n}},
$$

where the $\sigma_{i}$ are constants. For a given set of $\sigma_{i}, \bar{L}[u(x)]=0$ is an $m$ th order differential equation in the variable $x_{1}$, and there are $m$

- The index $\mu+j=\left(\mu_{1}+j, \mu_{2}, \cdots, \mu_{n}\right)$. 
independent solutions, $f\left(x_{1}\right)$. If we apply $\hat{L}$ to $u(x)$ as defined in equation (11), then for a given set of $\sigma_{i}$ we get either at most $m-1$ independent solutions for $f\left(x_{1}\right)$ or that the coefficients of all derivatives of $f\left(x_{1}\right)$ in $\hat{L}$ vanish.

Since equation (10) is to hold for all solutions $u$ of $\bar{L}[u]=0$, it is impossible for $\hat{L}[u]=0$ to give only $m-1$ solutions for $f\left(x_{1}\right)$. On the other hand, if the coefficients of all orders of $\partial / \partial x_{1}$ vanish in equation (10) for all sets of $\sigma_{i}$, they vanish identically. We therefore conclude that $J_{s}=J_{s}^{\prime}$, i.e., equation (5) is unique.

We now apply identity (5) to $u=K(y-z)$, which satisfies $\bar{L}_{y}[K(y-z)]=0$ for $z \neq y$, since $L$ is self-adjoint. Substituting in equation (4) we get the normalized representation:

$$
v(z)=\omega_{1}(z)-\sum_{v=0}^{\infty} \sum_{|s|=p ; s_{1}<m} J_{s} D_{y}^{0} K(y-z) \quad \text { for } z \neq y, z \in D
$$

where we have defined $A_{\mathrm{s}}=J_{\mathrm{s}}$ for $|s|<m$.

We now prove that equation (12) is a unique representation of $v(z) .{ }^{7}$ In particular it is independent of the construction leading to the coefficients $A_{i}$. For suppose we had another representation of $v(z)$ :

$$
v(z)=\omega_{2}(z)-\sum_{v=0}^{\infty} \sum_{|s|=v_{i} s_{1}<m} J_{\imath}^{\prime} D_{y}^{\prime} K(y-z), \quad \text { for } z \neq y, z \in D^{\prime},
$$

where $D^{\prime}$ is some annular region about the point $x=y$ such that $D \cap D^{\prime}=D^{\prime \prime}$ is a nonempty set. Let the outer ball of $D^{\prime \prime}$ be $B_{0}^{\prime \prime}$ with radius $r_{0}$, and the inner ball be $B_{I}^{\prime \prime}$ with radius $r_{I}$.

Subtracting equation (13) from equation (12) we get for $z \neq y$, $z \in D^{\prime \prime}$ :

$$
\phi(z)=\omega_{1}(z)-\omega_{2}(z)=\sum_{\nu=0}^{\infty} \sum_{|z|=\nu ; s_{1}<m}\left(J_{0}-J_{\imath}^{\prime}\right) D_{y}^{\bullet} K(y-z) .
$$

We want to prove that $J_{s}=J_{s}^{\prime}$ and $\omega_{1}(z)=\omega_{2}(z)$. We know the form of the fundamental solution $K(y-z) .^{8}$

$$
K(y-z)= \begin{cases}\rho^{m-n} A\left(\frac{z-y}{\rho}\right) & \text { for odd } n \\ \rho^{m-n}\left[B\left(\frac{z-y}{\rho}\right) \log \rho+C\left(\frac{z-y}{\rho}\right)\right] & \text { for even } n,\end{cases}
$$

\footnotetext{
${ }^{7}$ Unique within the choice of $x_{1}$

${ }^{8}$ See [1, pp. 298-303].
} 
where $\rho=|z-y|$, and $A, B, C, K(y-z)$ are analytic for all real $y, z$ with $y \neq z$. For $n$ even $\rho^{m-n} B((z-y) / \rho)$ is a polynomial in $z-y$. Then $D_{v}^{s} K(y-z)$ has the form:

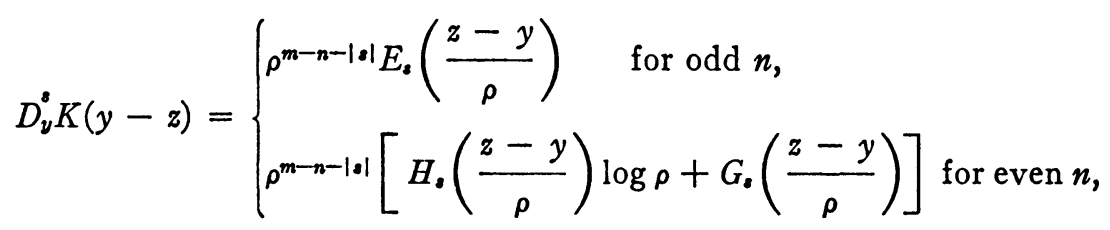

$E_{s}, H_{s}, G_{s}$ are regular for all real $z \neq y$. We can then write equation (14) in the form

$$
\begin{aligned}
\phi(z) & =\sum_{\nu=0}^{\infty} \sum_{|\varepsilon|=\nu ; s_{1}<m}\left(J_{s}-J_{s}^{\prime}\right) D_{y}^{s} K(y-z) \\
& =\sum_{\nu=0}^{\infty} \rho^{m-n-\nu} N_{\nu}\left(\frac{z-y}{\rho}\right)-Q\left(\rho, \frac{z-\nu}{\rho}\right) \log \rho .
\end{aligned}
$$

Let

$$
\eta=\frac{z-y}{\rho}
$$

then

$$
\phi(y+\rho \eta)=\sum_{\nu=0}^{\infty} \rho^{m-n-\nu} N_{\nu}(\eta)-Q(\rho, \eta) \log \rho,
$$

where $Q(\rho, \eta)$ is a polynomial in $z-y$ and $Q \equiv 0$ for odd $n$.

Consider an analytic continuation of equation (16) from the real $\rho$ to the complex $\zeta=\rho+i \xi$. For any fixed $\eta$, both $\phi(y+\zeta \eta)$ and $\sum_{\nu=0}^{\infty} \zeta^{m-n-\nu} N_{\nu}(\eta)$ are univalued functions, analytic in $\zeta$, and $Q(\zeta, \eta) \log \zeta$ is a multivalued function in $D^{\prime \prime}$. Since this is true for every fixed $\eta$, we conclude that for both odd and even $n$,

$$
\phi(y+\rho \eta)=\sum_{\nu=0}^{\infty} \rho^{m-n-\nu} N_{\nu}(\eta) .
$$

For any fixed $\eta$, the right-hand side of equation (17) converges not only for $r_{0}>\rho>r_{I}$ but for all $\infty>\rho \geqq r_{I}$. This is so because the series part of equation (17) becomes a power series in negative powers of $\rho$, except possibly for a finite number of positive powers in the case of $m-n>0 . \phi(y+\rho \eta)$ is then analytic for all $\infty>\rho \geqq 0$. From the analyticity of $\phi(y+\rho \eta)$ in $\infty>\rho \geqq 0$, we conclude, that the portion of the series in equation (17) containing negative powers of $\rho$ must vanish, i.e., 


$$
\sum_{\nu=0 ; m-n-\nu<0}^{\infty} \rho^{m-n-\nu} N_{\nu}(\eta)=0 .
$$

For $m-n<0$ this means that $\phi(z)=0$. When $m-n \geqq 0$ we get in addition:

$$
\sum_{\nu=0 ; m-n \geq 0}^{m-n} \rho^{m-n-\nu} N_{\nu}(\eta)=\phi(y+\rho \eta) .
$$

We will take these two cases separately. First for equation (19), using equation (15), we get:

$$
\phi(z)=\sum_{\nu=0 ; m-n \geq 0}^{m-n} \sum_{|s|=y}\left(J_{s}-J_{s}^{\prime}\right) D_{y}^{s} K(y-z)=L_{y}[K(y-z)]
$$

Since $|s| \leqq m-n$, from the form of $D_{v}^{s} K(y-z)$ we see that equation (20) holds even at $y=z$. Let $\bar{B}$ be a ball containing $y$ as an interior point. Let $\psi(\eta)$ be any regular function which vanishes in a neighborhood of $\bar{\beta}$, the boundary of $\bar{B}$. By Green's identity

$$
\psi(\eta)=\int_{\bar{B}} \bar{L}[\psi(z)] K(\eta-z) d z
$$

then

(21) $\left.\tilde{L}[\psi(\eta)]\right|_{\eta=y}=\tilde{L}_{y}[\psi(y)]=\int_{\bar{B}} \bar{L}[\psi(z)] \phi(z) d z=\int_{\bar{B}} \psi(z) \bar{L}[\phi(z)] d z$.

Since $L$ is self-adjoint and $\phi$ is a solution of $\bar{L}[\phi]=0$, equation (21) implies that every regular function $\psi$, which vanishes in a neighborhood of $\bar{\beta}$ has the property that at $x=y, \tilde{L}[\psi]=0$. We then conclude that $\tilde{L} \equiv 0$ and therefore from equation $(20) \phi \equiv 0$, i.e., $\omega_{1}(z)=\omega_{2}(z)$. Also for $m-n \geqq 0$, and $m-n \geqq|s| \geqq 0$, we have $J_{s}=J_{s}^{\prime}$.

We now return to equation (18). Since for every fixed $\eta$ equation (18) is a power series its terms vanish separately. From equation (15) this means that:

$$
\sum_{|\varepsilon|=p ; m-n-\nu<0}\left(J_{\bullet}-J_{\imath}^{\prime}\right) D_{y}^{z} K(y-z)=0 .
$$

This could be written:

$$
\begin{aligned}
\sum_{|s|=>; m-n-r<0}\left(J_{s}-J_{s}^{\prime}\right) D_{y}^{8} K(y-z) & = \pm \sum_{|s|=v ; m-n-r<0}\left(J_{z}-J_{s}^{\prime}\right) D_{z}^{8} K(y-z) \\
& =P_{z}[K(y-z)]=0 .
\end{aligned}
$$


$P_{z}$ is a linear homogeneous operator with constant coefficients, and equation (23) holds for all $z$ such that $r_{I} \leqq|y-z|$. But $K(y-z)$ is analytic for all real $z$ with $z \neq y$. Then equation (21) holds for all real $z$ with $z \neq y$.

Let $G$ be a simply connected open finite region. Let $\beta_{G}$ be the boundary of $G$. Then a regular solution $f$, of $\bar{L}[f]=0$ has a representation in $G$ :

$$
f(z)=\int_{\beta_{G}} M[K(\xi-z), f(\xi)] d S_{\xi}
$$

and so

$$
P_{z}[f(z)]=\int_{\beta_{G}} M\left[P_{z}[K(\xi-z)], f(\xi)\right] d S_{\xi}=0 \text { in } G .
$$

Then every regular solution $f$ of $\bar{L}[f]=0$ in $G$ is also a solution of $P_{z}[f(z)]=0$ in $G$. But $P_{z}$ has the property that all $D_{z}^{s}$ in $P_{z}$ have $s_{1} \leqq m-1$. It has been proved previously that not every $f(z)$ which solves $\bar{L}_{z}[f(z)]=0$ can solve $P_{z}[f(z)]=0$ unless $P_{z} \equiv 0$. We then conclude that $J_{\mathrm{s}}=J_{\text {? }}^{\prime}$ for all $|s| \geqq 0$, and that equation (12) is a unique representation of equation (2). Furthermore, this representation holds right up to the singularity, since in our original construction we may now take $r_{2}>0$, the radius of $S_{2}$ as small as we wish.

\section{BIBLIOGRAPHY}

1. Fritz John, The fundamental solution of linear elliptic differential equations with analytic coefficients, Comm. Pure Appl. Math. 3 (1950), 273-304.

2. R. Courant and D. Hilbert, Methods of mathematical physics, Vol. I, Interscience, New York, 1953.

General Electric, King of Prussia, Pennsylvania 